\title{
ВЛИЯНИЕ МАГНИТНОГО ПОЛЯ НА КИНЕТИКУ ПЕРЕНОСА́ ЭЛЕКТРОНА МЕЖДУ ДИМЕРОМ ХЛОРОФИЛЛА И ФЕОФИТИНОМ В ФОТОСИНТЕТИЧЕСКИХ РЕАКЦИОННЫХ ЦЕНТРАХ
}

В реакционных центрах фотосинтезирующих бактерий за время $<10 n c$ после фотовозбуждения димера хлорофилла образуется радикальная пара (РП) $P_{s 0}{ }^{+} I^{-} \equiv P^{F}$ (обозначения см. на рис. 1), после чего за время $\simeq 0,2$ нс электрон переносится на первичный акцептор. Если первичный акцептор предварительно восстановлен химически, РП за время $\simeq 10 \mathrm{HC}$ рекомбинирует в основное состояние $P_{s 0 s 0} I$ и возбужденное триплетное состояние $P_{s 0 T} * I=P^{R}$, которое, в свою очередь, распадается в $P_{8080} I$ за время $\simeq 10$ мкс.

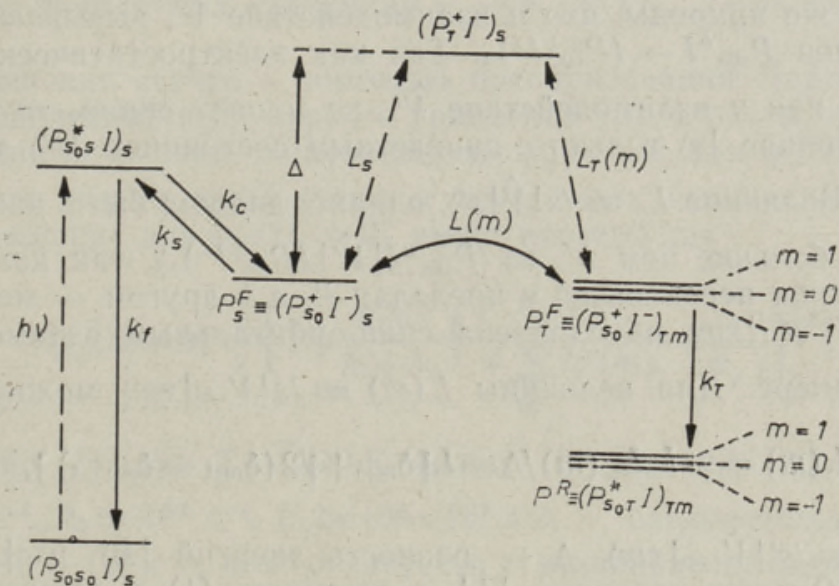

Рис. 1. Энергетическая схема первичных реакций бактериального фотосинтеза. $P$ - димер бактериохлорофилла, $I$ - бактериофеофитин. Основное синглетное, синглетное и триплетное состояния мономеров хлорофилла обозначены индексами $s_{0}, S$ и $T$. возле $P$ соответственно. Синглетное и триплетное состояния радикальной пары $P^{+} I^{-}$также обозначены индексами $s$ и $T$ соответственно. $k_{c}, k_{f}, k_{s}, k_{T}-$ константы скоростей соответствующих реакций. $L(m)-$ величнна эффективного резонансного взаимодействия.

Было обнаружено, что квантовый выход $\varphi_{T}$ триплетных состояний $P_{R}^{R}$ зависит от магнитного поля. Характер этой зависимости, а также аномальная спиновая поляризация спектров ЭПР триплетного состояния $P^{R}$ указывали на тесную аналогию с рядом других радикальных реакций, которые получили объяснение в рамках теории химической поляризации электронов и ядер (ХПЭия) (обзор экспериментальных результатов и теоретических подходов см. в $[1,2])$, 
Основной проблемой описания синглет-триплетных переходов в восстановленных реакционных центрах является определение взаимодействия, вызывающего переходы между синглетным $\left(P_{s 0}{ }^{+} I^{-}\right)_{s}$ и триплетным $\left(P_{s 0}{ }^{+} I^{-}\right)_{T}$ состояниями РП, которые затем рекомбинируют в $P_{s 0 s 0} I$ и $P^{R}$ соответственно. В теории ХПЭия $\left[{ }^{2}\right]$ такими являются зеемановское взаимодействие (если g-факторы радикалов пары различны) и сверхтонкое взаимодействие неспаренных электронов со своими магнитными ядрами.

Оказалось, однако, что разность g-факторов РП слишком мала, чтобы объяснить экспериментальные данные и, кроме того, было показано отсутствие магнитного изотопного эффекта $\left[{ }^{3,4}\right]$, хотя сверхтонкое взаимодействие сильно меняется при дейтерировании.

В настоящей работе мы предлагаем принципиально иной механизм синглет-триплетных переходов в РП $P^{+} I^{-}$, учитывающий димерную структуру пигмента $P$. Так, эффективное резонансное взаимодействие $\hat{V}_{e f f}$, вызывающее переходы между состоянием $|s\rangle \equiv\left|\left(P_{s 0^{+}}+I^{-}\right)_{s}\right\rangle$ и состоянием $|\mathrm{Tm}\rangle \equiv\left|\left(P_{s 0}^{+} I^{-}\right)_{\text {тm }}\right\rangle$, обусловлено примесью этих состояний к вышележащему виртуальному $\left|s^{\prime}\right\rangle \equiv\left|\left(P_{T}^{+} I^{-}\right)_{s}\right\rangle$. Состояние $\left|s^{\prime}\right\rangle$ может быть получено из $|s\rangle$ в результате электронного переноса в пределах $P^{+}$, когда один из мономеров $P^{+}$окисляется в основном синглетном состоянии, а другой $P^{+}$восстанавливается в возбужденном триплетном состоянии. Әтот перенос обусловлен электростатическим взаимодействием $\hat{V}$ той же природы, что и взаимодействие $\hat{V}^{\prime}$, вызывающее разделение зарядов $P_{s 0 s}{ }^{*} I \rightarrow\left(P_{s 0}+I^{-}\right)_{s}$. Так как электростатическое взаимодействие $\hat{V}$, как и взаимодействие $\hat{V}^{\prime}$, не меняет спина. то оно может связать состояние $|s\rangle$ только с синглетным состоянием $\left|s^{\prime}\right\rangle$ другой конфигурации. Величина $L_{s} \equiv\left\langle s|\hat{V}| s^{\prime}\right\rangle$, однако, должна быть, как минимум, на порядок больше, чем $L_{s}^{\prime} \equiv\left\langle P_{s 0 s}{ }^{*} I\left|\hat{V}^{\prime}\right|\left(P_{s 0}{ }^{+} I^{-}\right)_{s}\right\rangle$, так как в первом случае электрон переносится в пределах $P$. а в другом - между $P$ и $I$. Примесь $\left|s^{\prime}\right\rangle$ к $|T m\rangle$ обусловлена спин-орбитальным взаимодействием $\hat{V}_{s-0}$ в мономере. Для величины $L(m) \equiv\left\langle s\left|\hat{V}_{\text {eff }}\right| T m\right\rangle$ можно получить

$$
L(m)=-L_{s} L_{\tau}(m) / \Delta=L\left[\delta_{m ; 0}+\sqrt{2}\left(\delta_{m ; 1}-\delta_{m ;-1}\right)\right],
$$

где $L_{T}(m) \equiv\left\langle s^{\prime}\left|\hat{V}_{s-0}\right| T m\right\rangle, \Delta-$ разность энергий $\left|s^{\prime}\right\rangle$ и $|s\rangle$, равная $\simeq 10^{4} \mathrm{~cm}^{-1}$. Оценим величину $|L|$ с помощью (1) и выражения $k_{i} \simeq$ $\simeq 4 L_{i}^{2} / x$ для скорости резонансного перехода на уровень, релаксирующий с константой $x$. Предположим, что релаксащия обvсловлена внутренней конверсией $в$ терме $\left(x \simeq 10^{12}-10^{13} c^{-1}\right)$. Поскольку $k\left(P^{*} \rightarrow I^{-}\right) \simeq 10^{11} c^{-1}$. то $\left|L_{s}^{\prime}\right| \simeq \sqrt{k\left(P^{*} \rightarrow I^{-}\right) x} \simeq 10^{11}-10^{12} c^{-1}$ и $\left|L_{s}\right| \simeq$ $\simeq 10^{12}-10^{13} \mathrm{c}^{-1}$. Скорость $k\left(T \rightarrow S_{0}\right)$ для мономера бактериохлорофилла составляет $\simeq 10^{4}-10^{5} c^{-1}$ и, следовательно, $\left|L_{T}(m)\right| \simeq \sqrt{k\left(T \rightarrow s_{0}\right) x} \simeq$ $\simeq 10^{8}-10^{9} c^{-1}$. В итоге получаем, что $|L| \simeq 10^{7}-10^{8} c^{-1}$. Существенно то, что $L$ не меняется при дейтерировании.

Таким образом, для описания эволющии спинов РП мы предлагаем использовать гамильтониан

$$
H_{0}=g \beta_{e} H \sum_{m} m|T m\rangle\langle T m| f \sum_{m} L(m)(|s\rangle\langle T m|+| T m\rangle\langle s|) .
$$

Запишем кинетические уравнения для спиновой матрицы плотности $\hat{\mathrm{Q}}(t)$ системы, изображенной на рис, 1, Так как волновые функции 
состояний $|s\rangle$ и $|T m\rangle$ отличаются только спиновой частью, вклад в релаксацию недиагональных элементов матрицы плотности $\hat{\mathrm{Q}}(t)$ будут давать только процессы гибели этих состояний с временами $k_{s}^{-1}$ и $k_{\mathrm{\tau}}^{-1}$. Учитывая (2) и заменяя везде далее $|\mathrm{Tm}\rangle$ на $|m\rangle$, можно получить

$$
\begin{aligned}
& \dot{\varrho}_{s s}=\sum_{m} L(m) x_{m}-k_{s @ s s}+k_{c \varrho s * s^{*}}, \quad \dot{\varrho s s}(t=0)=1 \text {, } \\
& \dot{\mathrm{Q} m m}=-L(m) x_{m}-k_{T} \mathrm{Qmm},
\end{aligned}
$$

$$
\begin{aligned}
& \dot{x}_{m}=-m h y_{m}+2 L(m)\left(\varrho_{m m}-\varrho_{s s}\right)+\sum_{l(\neq m)} L(l)\left(\varrho_{m l}+\mathrm{Q}_{l m}\right)-\left(k_{s}+k_{T}\right) x_{m} / 2 \text {, } \\
& \dot{y}_{m}=m h x_{m}+i \sum_{u \neq m)} L(l)(\varrho l m-\varrho m l)-\left(k_{s}+k_{T}\right) y_{m} / 2, \\
& \dot{\mathrm{Q} m l}=i h(m-l) \mathrm{Qml}+i(L(m) \mathrm{Q} s l-L(l) \mathrm{gms})-k_{\tau} \mathrm{\varrho} m l, . \\
& \dot{\varrho}_{s^{*} s^{*}}=-\left(k_{c}+k_{f}\right) \varrho_{s^{*} s^{*}}+k_{s} \varrho_{s s}
\end{aligned}
$$

где $\quad x_{m}=i(\varrho m s-\varrho s m), y_{m} \equiv \varrho m s f \varrho s m, \quad m, l=0,+1,-1,\left|s^{*}\right\rangle=\left|P_{s 0 s}^{*} I\right\rangle$, $h=g \beta_{e} H$.

Решить систему уравнений (3), (4) нам не удается, однако квантовый выход триплетных состояний $\varphi_{T}=k_{T} \sum_{m} \int_{0}^{\infty} \varrho m m(t) d t$ можно определить совершенно строго с помощью преобразования Лапласа, сначала отбросив последний член первого уравнения (3), а затем учтя ёго методом функции источника. Получающееся при этом для $\varphi_{\tau}(H)$ выражение оказывается весьма громоздким и неудобным для анализа. В то же время выражение для $\varphi_{T}(H=0)$ имеет простой вид

$$
\varphi_{T}(H=0)=\left[1+\frac{k_{f} k_{s}}{k_{c}+k_{f}}\left(\frac{k_{T}+k_{s}}{4 \sum_{m} L^{2}(m)}+\frac{1}{k_{T}}\right)\right]^{-1} .
$$

Согласно [ $\left.{ }^{1}\right]$, при $T=300 \mathrm{~K}$ имеем . $\varphi_{\tau}(0)=0,1-0,2, k_{c} \simeq 10^{11} c^{-1}$, $k_{f_{1}} \simeq 10^{10} c^{-1}, k_{s} \simeq 10^{9} c^{-1}, k_{\tau} \simeq 10^{8}-10^{9} c^{-1}$, следовательно, $k_{r}, k_{s} \gg$ $\gg|L| \simeq 10^{7}-10^{8} c^{-1}$, что согласуется с вышеприведенными качественными оценками. Это позволяет оставить в (4) только линейные по $|L|$ члены и получить простое выражение для

$$
R(H) \equiv \frac{\varphi(H)}{\varphi_{T}(0)}=R(\infty)+(1-R(\infty))\left[1+\left(H / H_{1 / 2}\right)^{2}\right]^{-1}
$$

где

$$
\begin{gathered}
R(\infty)=0,2 \cdot \frac{20 L^{2}\left(k_{c}+k_{f}\right)+k_{f} k_{s}\left(k_{r}+k_{s}\right)}{4 L^{2}\left(k_{c}+k_{f}\right)+k_{f} k_{s}\left(k_{T}+k_{s}\right)}, \\
H_{1 / \mathrm{s}}=\frac{k_{T}+k_{s}}{2 g \beta_{e}} \sqrt{5 R(\infty)}
\end{gathered}
$$

- магнитное поле, при котором $R(H)=(1+R(\infty)) / 2$.

Зависимость $R(H)$ (6) (см. рис. 2) описывает качественные особенности экспериментальных кривых $\left[{ }^{1}\right]$, однако отсутствие подробных 


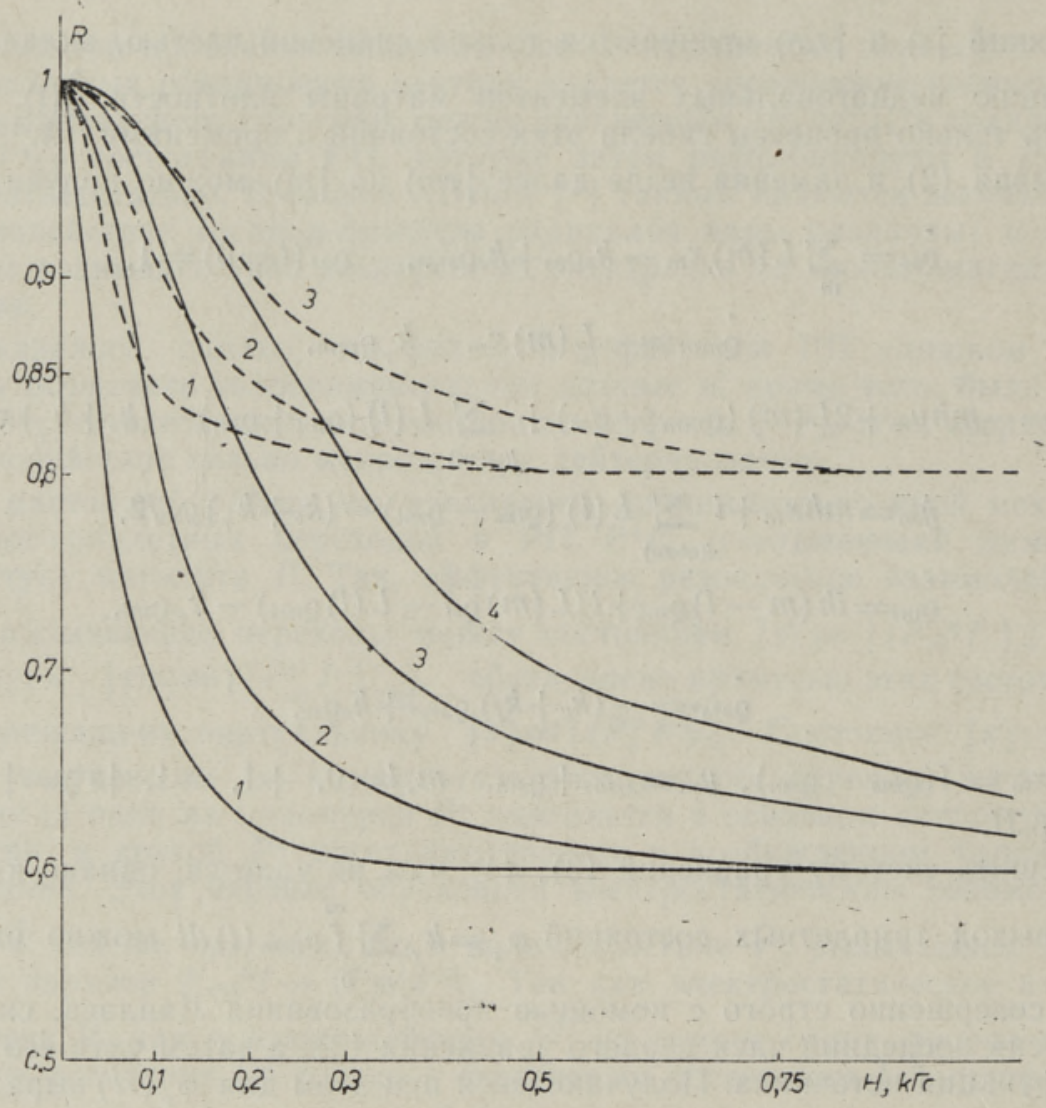

Рис. 2. Зависимость $R(H)$, построенная по формуле (6), для $R(\infty)=$ $=0,6$ ( $), 0,8(--\longrightarrow)$ и $\mathrm{H}_{1 / 2}=0,05$ (1), $0,1 \quad(2), 0,2$ (3) и

экспериментальных данных не позволяет делать более конкретных выводов. При наличии же таких данных можно будет, не прибегая к теории возмущений по $|L|$, находить точные значения $\varphi_{\tau}(H)$, решая систему линейных алгебраических уравнений для Лаплас-образов элементов матрицы плотности.

\section{ЛИТ Е РА Т У РА}

1. Hof f, A. I., Phys. Reports, 54, № 2, 75-200 (1979).

2. Буч а че н ко А. Л., С агде ев Р. З., Салихов К. М., Магнитные и спиновые эффекты в химических реакциях, Новосибирск, «Наука», 1978.

3. R a demaker, H., Hoff, A. I., van Grondelle, R., D uysens, L. N. M. Biochim. Biophys. Acta, 592, 240-257 (1980).

4. B la nkenship, R. E., Parson, W. W., Biophys. J., 25, 205a (1979).

Институт теоретической физики Академии наук Украинской ССР 


\section{MAGNETVÃLJA MOJU KLOROFULLI DIMEERI JA FEOFUTIINI VAHELISE ELEKTRONIULEKANDE KINEETIKALE FOTOSONTEETILISES REAKTSIOONITSENTRIS}

On esitatud klorofülli dimeeri singlett-triplettsiirde uus mehhanism fotosünteesivate bakterite keemiliselt taastatud reaktsioonitsentrites. On saadud lihtne avaldis, mis väljendab dimeeri trip!ettolekute kvantsaagise sõltuvust magnetväljast.

A. A. DEMIDENKO, E. G. PETROV

INFLUENCE OF THE MAGNETIC FIELD ON THE KINETICS OF THE ELECTRON TRANSFER BETWEEN THE CHLOROPHYLL DIMER AND THE PHEOPHYTIN IN PHOTOSYNTHETIC REACTION CENTRES

A new mechanism is proposed to explain the singlet-triplet transitions in the chlorophyll dimer of chemically-reduced photosynthetic reaction centres. A simple expression for the magnetic field dependence on the triplet quantum yield is obtained. 